\title{
THE SQ-UNIVERSALITY OF ONE-RELATOR RELATIVE PRESENTATIONS
}

\author{
Anton A. Klyachko \\ Faculty of Mechanics and Mathematics, Moscow State University \\ Moscow 119992, Leninskie gory, MSU \\ klyachko@daniil.math.msu.su
}

\begin{abstract}
Adding two generators and one arbitrary relator to a nontrivial torsion-free group, we always obtain an SQ-universal group. In the course of the proof of this theorem, we obtain some other results of independent interest. For instance, adding one generator and one relator in which the exponent sum of the additional generator is one to a free product of two nontrivial torsion-free groups, we also obtain an SQ-universal group.

Key words: relative presentations, one-relator groups, SQ-universality, equations over groups.

$M S C: 20 \mathrm{E} 06,20 \mathrm{~F} 05,20 \mathrm{~F} 06$.
\end{abstract}

\section{Introduction}

Recall that a group $G$ is called $S Q$-universal if every countable group can be embedded in some quotient of $G$. Examples of SQ-universal groups are all nonabelian free groups; all free products, except the infinite dihedral group $\mathbb{Z}_{2} * \mathbb{Z}_{2}$ (see [LS77]); many amalgamated free products and HNN-extensions ([Lo86], [LS77]); all finite-index subgroups of SQ-universal groups and all virtually SQ-universal groups [Neu73]; all nonelementary hyperbolic groups [Ols95] and even all (with some obvious exceptions) relatively hyperbolic groups, in particular, all groups with infinitely many ends [AMO06].

The starting point for our study is the following theorem.

Sacerdote-Schupp theorem [SaSc74] (see also [LS77]). A group admitting a presentation with one relator and at least three generators is $S Q$-universal.

Actually, there is a much more general fact.

Baumslag-Pride theorem [BaPr78]. A group having a presentation with two more generators than relators is SQuniversal. Moreover, any such group is large in the sense of Gromov, i.e. it has a finite-index subgroup admitting an epimorphism onto a nonabelian free group.

A further generalisation of the Sacerdote-Schupp theorem is the following result.

Stöhr-Gromov theorem [St83], [Gr83]. A group having a presentation in which there are more generators than relators and one of the relators is a proper power is SQ-universal and even large in the sense of Gromov.*)

Further results on this subject can be found in, e.g., [Ed84], [How98], [Bu05], [La05], [OlOs06]. In this paper, we generalise the Sacerdote-Schupp theorem in another direction.

Let $G$ be a group. A group given by a one-relator relative presentation over $G$ is

$$
\widetilde{G}=\left\langle G, x_{1}, x_{2}, \ldots, x_{n} \mid w=1\right\rangle \stackrel{\text { def }}{=} G * F\left(x_{1}, x_{2}, \ldots, x_{n}\right) /\langle\langle w\rangle\rangle .
$$

Here $x_{1}, \ldots, x_{n}$ are some letters (not belonging to $G$ ) and $w$ is a word in the alphabet $G \cup\left\{x_{1}^{ \pm 1}, \ldots, x_{n}^{ \pm 1}\right\}$ (such a word can be considered as an element of the free product $G * F\left(x_{1}, x_{2}, \ldots, x_{n}\right)$ of $G$ and the free group with basis $\left.x_{1}, x_{2}, \ldots, x_{n}\right)$. In other words, the presentation of the group $\widetilde{G}$ is obtained from a presentation $G=\langle A \mid R\rangle$ of $G$ by adding several new generators and one new relator: $\widetilde{G}=\left\langle A \cup\left\{x_{1}, x_{2}, \ldots, x_{n}\right\} \mid R \cup\{w\}\right\rangle$.

Theorem 1. If $G$ is a nontrivial torsion-free group and $n \geqslant 2$, then the group $\widetilde{G}=\left\langle G, x_{1}, x_{2}, \ldots, x_{n} \mid w=1\right\rangle$ is $S Q$-universal for any $w \in G * F\left(x_{1}, \ldots, x_{n}\right)$.

Corollary [Kl06b]. Under the conditions of Theorem 1 the group $\widetilde{G}$ (as well as any SQ-universal group) has a nonabelian free subgroup.

Remark 1. It is easy to show that the group $\widetilde{G}$ from Theorem 1 need not be large in the sense of Gromov.

Remark 2. Certainly, the assertion of Theorem 1 is not valid for $n=1$ (as well as the assertion of the SacerdoteSchupp theorem is not valid for groups with two generators). However, the group $\widetilde{G}$ with $n=1$ has some properties weaker than the SQ-universality. In particular, $\widetilde{G}$ is nontrivial [K193], it cannot be a nonabelian simple group (if

This work was supported by the Russian Foundation for Basic Research, project no. 05-01-00895.

*) A proper power is an element of a free group $F$ of the form $u^{k}$, where $u \in F$ and $\mathbb{Z} \ni k \geqslant 2$. In particular, the identity element is a proper power. Thus, the Stöhr-Gromov theorem is a generalisation of the Baumslag-Pride theorem. 
$w \neq g_{1} x_{1}^{ \pm 1} g_{2}$ ) [K105], and the natural mapping $G \rightarrow \widetilde{G}$ is nonsurjective (if $w \neq g_{1} x_{1}^{ \pm 1} g_{2}$ ) [CR01]; in the case when the exponent sum of $x_{1}$ in $w$ is \pm 1 , the group $\widetilde{G}$ always (with some obvious exceptions) contains a nonabelian free subgroup [Kl06b].

Theorem 1 considers relative presentations with at least two additional generators; however, an important role in the proof of this theorem is played by the study of one-generator relative presentations

$$
\left.\widetilde{G}=\langle G, t \mid w=1\rangle \stackrel{\text { def }}{=}\left(G *\langle t\rangle_{\infty}\right) /\langle w\rangle\right\rangle, \quad \text { where } w \equiv \prod g_{i} t^{\varepsilon_{i}}, g_{i} \in G, \varepsilon_{i} \in\{ \pm 1\} .
$$

Such a presentation is called unimodular if $\sum \varepsilon_{i}= \pm 1$. It is known that unimodular relative presentations have some good properties and are more convenient to study (see, e.g., [K193], [Kl94], [FeR96], [CR01], [FoR05], [K105], [Kl06a], $[\mathrm{K} 106 \mathrm{~b}])$.

In [Kl06a], we suggested a generalisation of the notion of unimodularity to the so-called generalised relative presentation

$$
\widetilde{G}=\left\langle G * T \mid \prod_{i=1}^{n} g_{i} t_{i}=1\right\rangle \stackrel{\text { def }}{=}(G * T) /\left\langle\left\langle\prod g_{i} t_{i}\right\rangle\right\rangle .
$$

Here $T$ is a group (not necessarily cyclic), $g_{i} \in G$, and $t_{i} \in T$; the word $\prod_{i=1}^{n} g_{i} t_{i}$ is assumed to be cyclically reduced.

The generalised relative presentation $(*)$ over a group $G$ is called unimodular if

1) the order of the element $\prod t_{i}$ is infinite in the group $T$;

2) the cyclic subgroup $\left\langle\prod t_{i}\right\rangle$ is normal in $T$;

3) the quotient group $T /\left\langle\prod t_{i}\right\rangle$ possesses the strong unique-product property.

Recall that a group $H$ is called a UP-group, or a group with the unique product property, if the product $X Y$ of any two finite nonempty subsets $X, Y \subseteq H$ contains at least one element which decomposes uniquely into the product of an element from $X$ and an element from $\left.Y{ }^{*}\right)$

We say that a group $H$ has the strong unique product property if the product $X Y$ of any two finite nonempty subsets $X, Y \subseteq H$ such that $|Y| \geqslant 2$ contains at least two uniquely decomposable elements $x_{1} y_{1}$ and $x_{2} y_{2}$ such that $x_{1}, x_{2} \in X, y_{1}, y_{2} \in Y$, and $y_{1} \neq y_{2}$.

As far as we know, all known examples of UP-groups have the strong UP-property. In particular, all right orderable groups, locally indicable groups, and diffuse groups in the sense of Bowditch have the strong UP property.

Theorem 1 is an easy corollary of the following theorem.

Theorem 2. If the generalised relative presentation $(*)$ over a noncyclic torsion-free group $G$ is unimodular and the group $T$ is not cyclic, then the group $\widetilde{G}$ given by presentation (*) is $S Q$-universal.

In order to prove Theorem 2, we establish the following fact about usual (nongeneralised) unimodular relative presentations.

Theorem 3. If $G_{1}, \ldots, G_{l}$ are noncyclic torsion-free groups, $l \geqslant 2$, and a relative presentation

$$
L=\left\langle G_{1} * \ldots * G_{l}, t \mid w=1\right\rangle
$$

over the group $G_{1} * \ldots * G_{l}$ is unimodular, then the group $L$ is $S Q$-universal. Moreover, each countable group $S$ embeds into a quotient group $L / N$, in which the Freiheitssatz holds, i.e.,

$$
\left\langle t, G_{i_{1}}, \ldots, G_{i_{l-1}}\right\rangle=\langle t\rangle_{\infty} * G_{i_{1}} * \ldots * G_{i_{l-1}} \quad \text { in } L / N
$$

if the word $w$ is conjugate in group $\langle t\rangle_{\infty} * G_{1} * \ldots * G_{l}$ to no element of the subgroup $\langle t\rangle_{\infty} * G_{i_{1}} * \ldots * G_{i_{l-1}}$.

According to [K106a], we say that presentation (1) is magnusian if the natural mapping $G \rightarrow \widetilde{G}$ is injective and $\langle H, t\rangle=H *\langle t\rangle_{\infty}$ in the group $\widetilde{G}$ (i.e., $t$ is transcendental over $H$ in $\widetilde{G}$ ) for any free factor $H$ of $G$ such that $w$ is not conjugate in $G *\langle t\rangle_{\infty}$ to an element of $H *\langle t\rangle$.

In [Kl06a], we showed that every unimodular presentation over a torsion-free group is magnusian. To prove the main results of this paper, we need a stronger property of unimodular presentations.

We say that presentation (1) is strongly magnusian if the element $t$ is transcendental in $\widetilde{G}$ over each subgroup $H \subseteq G$ such that

1) $w$ is not conjugate in $G *\langle t\rangle_{\infty}$ to an element of $H *\langle t\rangle$;

2) each coefficient $g_{i}$ either lies in $H$ or is transcendental over $H$.

*) Some time ago, there was the conjecture that any torsion-free group is UP (the converse is, obviously, true). However, it turned out that there exist counterexamples ([P88], [RS87]). 
Proposition 1. If presentation (1) is unimodular and each nonidentity coefficient $g_{i}$ has infinite order in $G$, then presentation (1) is strongly magnusian.

The proof of Proposition 1 is based on several earlier known results, in particular, on the following theorem.

Theorem 4. Suppose that $A * B$ is the free amalgamated product of groups $A$ and $B$ with amalgamated subgroup $C$, $v=b_{0} a_{0} \ldots b_{m} a_{m} b_{m+1} \in(A * B), m \geqslant 1$, and each coefficient of the word $v$ (except maybe the first and the last ones) is transcendental over $C$, i.e., $\left\langle a_{i}, C\right\rangle=\left\langle a_{i}\right\rangle_{\infty} * C$ in $A$ for $i=0, \ldots, m$ and $\left\langle b_{i}, C\right\rangle=\left\langle b_{i}\right\rangle_{\infty} * C$ in $B$ for $i=1, \ldots, m$. Then, for any automorphism $\varphi$ of the group $B$, the natural mappings

$$
A \rightarrow\left\langle\underset{C}{\underset{C}{*}} B \mid\left\{b^{v}=b^{\varphi} \mid b \in B\right\}\right\rangle \leftarrow B
$$

are injective.

This theorem was proven in [K194], but it has never been published. The last section of this paper contains a proof of Theorem 4. Contrary to purely algebraic arguments of all preceding sections, the proof of Theorem 4 is geometric. Other known facts from which Proposition 1 is derived have similar proofs.

Notation which we use is mainly standard. Note only that if $k \in \mathbb{Z}, x$ and $y$ are elements of a group, and $\varphi$ is a homomorphism from this group into another group, then $x^{y}, x^{k y}, x^{-y}, x^{\varphi}, x^{k \varphi}$, and $x^{-\varphi}$ denote $y^{-1} x y, y^{-1} x^{k} y$, $y^{-1} x^{-1} y, \varphi(x), \varphi\left(x^{k}\right)$, and $\varphi\left(x^{-1}\right)$, respectively; the commutator $[x, y]$ is understood as $x^{-1} y^{-1} x y$. If $X$ is a subset of a group, then $\langle X\rangle$ and $\langle\langle X\rangle\rangle$ denote the subgroup generated by $X$ and the normal subgroup generated by the set $X$, respectively. The symbol $|X|$ denotes the cardinality of the set $X$.

\section{Proof of Theorem 1}

If the group $G$ is cyclic, then $\widetilde{G}$ is a group with at least three generators and one relator. The SQ-universality of such groups is asserted by the Sacerdote-Schupp theorem. Thus, we assume that $G$ is noncyclic.

Suppose that the word $w$ has the form $w \equiv g_{1} x_{j_{1}}^{\varepsilon_{1}} g_{2} x_{j_{2}}^{\varepsilon_{2}} \ldots g_{p} x_{j_{p}}^{\varepsilon_{p}}$ and the word $w^{\prime} \in F\left(x_{1}, \ldots, x_{n}\right)$ is obtained from $w$ by erasing the coefficients: $w^{\prime}=x_{j_{1}}^{\varepsilon_{1}} x_{j_{2}}^{\varepsilon_{2}} \ldots x_{j_{p}}^{\varepsilon_{p}}$.

Case 1: $w^{\prime}$ is a proper power in the free group $F\left(x_{1}, \ldots, x_{n}\right)$. In this case, the group $\widetilde{G}$ is SQ-universal, because the one-relator homomorphic image $T_{1}=\left\langle x_{1}, \ldots, x_{n} \mid w^{\prime}=1\right\rangle$ of $\widetilde{G}$ is SQ-universal by the Stöhr-Gromov theorem.

Case 2: $w^{\prime}$ is not a proper power. Consider the groups

$$
T=\left\langle x_{1}, \ldots, x_{n} \mid\left[x_{1}, w^{\prime}\right]=\ldots=\left[x_{n}, w^{\prime}\right]=1\right\rangle \quad \text { and } \quad T_{1}=\left\langle x_{1}, \ldots, x_{n} \mid w^{\prime}=1\right\rangle=T /\left\langle w^{\prime}\right\rangle .
$$

The group $T$ is the free central extension of the one-relator group $T_{1}$. It is well known that if $w^{\prime}$ is not a proper power in the free group $F\left(x_{1}, \ldots, x_{n}\right)$, then the group $T_{1}$ is locally indicable ([B84]) and, therefore, has strong unique-product property. The element $w^{\prime}$ has infinite order in $T$ (see [LS77]). Thus, the generalised relative presentation $\langle G, T \mid w=1\rangle$ is unimodular. The group $T$ is not cyclic, because its commutator quotient is the free abelian group of rank $n \geqslant 2$. Therefore, by Theorem 2, the group $\langle G, T \mid w=1\rangle$ is SQ-universal. It remains to note that this group is a homomorphic image of $\widetilde{G}$, and a group having SQ-universal homomorphic image is SQ-universal itself.

\section{Iterated amalgamated free products}

In this section, we reproduce a construction from [Kl06a] in a more general situation.

Let $I$ be a set, and let $\Omega$ be a family of subsets of $I$. For each $i \in I$, let $G_{i}$ be a group, and for each $\omega \in \Omega$, let $G_{\omega}$ be a quotient of the free product $\underset{i \in \omega}{*} G_{i}$ :

$$
G_{\omega}=\left(\underset{i \in \omega}{*} G_{i}\right) / N_{\omega}
$$

The natural question arises: under what conditions are the natural mappings

$$
\varphi_{\omega}: G_{\omega} \rightarrow G_{I} \stackrel{\text { def }}{=}\left(\underset{i \in I}{*} G_{i}\right) /\left\langle\left\langle\bigcup_{\omega \in \Omega} N_{\omega}\right\rangle\right)
$$

injective? Or under what conditions can the group $G_{I}$ be considered as an amalgamated free product of the groups $G_{\omega}$ ?

The following proposition gives some sufficient condition for this question to have a positive answer. 
Proposition 2. Suppose that

$$
N_{\omega} \cap \underset{j \in \omega \backslash\{i\}}{*} G_{j}=\{1\}
$$

for each $\omega \in \Omega$ and each $i \in \omega \backslash(\bigcap \Omega)$. Suppose also that, for each finite subfamily $F \subseteq \Omega$ with $|F| \geqslant 2$, there exist elements min, $\max \in \bigcup F$ such that

1) the element min belongs to precisely one set $\omega_{\min } \in F$;

2) the element max belongs to precisely one set $\omega_{\max } \in F$;

3) $\omega_{\min } \neq \omega_{\max }$.

Then all of the natural mappings $\varphi_{\omega}: G_{\omega} \rightarrow G_{I}$ are injective.

Example. Suppose that $I=\{a, b, c, d, e, f\}$ and $\Omega=\{\{a, b, d, e\},\{b, c, e, f\},\{d, e, f\}\}$.

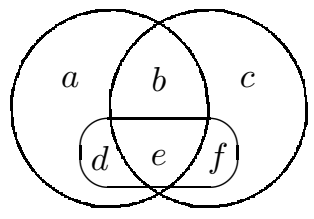

Let $A, \ldots, F$ be the corresponding six groups $G_{i}$, and let ABDE, BCEF, and DEF be the three groups $G_{\omega}$. It is easy to see that conditions 1), 2), and 3) hold for the family $\Omega$ and each of its two-set subfamilies. Suppose that condition $(* *)$ holds too. Then the validity of Proposition 2 (for this example) is implied by the following decomposition of $G_{I}$ into an amalgamated free product:

$$
G_{I}=((\mathbf{D E F} * B) \underset{B * D * E}{*} \mathbf{A B D E}) \underset{B * E * F}{*} \mathbf{B C E F}
$$

To prove Proposition 2 in the general case, we need a lemma.

Lemma 1. Suppose that the conditions of Proposition 2 hold, $\Omega^{\prime}$ is a finite subfamily of $\Omega, \omega \in \Omega$, and $\alpha \subseteq \omega \cap\left(\bigcup \Omega^{\prime}\right)$ is a proper subset of $\omega$ contained in $\bigcup \Omega^{\prime}$ and containing $\bigcap \Omega$. Then the natural mapping

$$
\underset{i \in \alpha}{*} G_{i} \rightarrow G_{\Omega^{\prime}} \stackrel{\text { def }}{=}\left(\begin{array}{c}
* \\
* \\
* \in \Omega^{\prime}
\end{array} G_{i}\right) /\left\langle\left\langle\bigcup_{\omega^{\prime} \in \Omega^{\prime}} N_{\omega^{\prime}}\right\rangle\right)
$$

is injective.

\section{Proof.}

Case 1: $\omega \in \Omega^{\prime}$. Let us use induction on the cardinality of $\Omega^{\prime}$. If $\left|\Omega^{\prime}\right|=1$ (i.e., $\Omega^{\prime}=\{\omega\}$ ), then the assertion of Lemma 1 is true by condition (**). Suppose that $\left|\Omega^{\prime}\right| \geqslant 2$. In this case, according to conditions 1$\left.), 2\right)$, and 3 ), the family $F=\Omega^{\prime}$ contains a set $\omega^{\prime} \neq \omega$ that contains an element $m \in \omega^{\prime}$ not lying in $\bigcup\left(\Omega^{\prime} \backslash\left\{\omega^{\prime}\right\}\right)$.

By the induction hypothesis (applied to the set $\omega^{\prime}$ as $\omega$ and the family $\Omega^{\prime} \backslash\left\{\omega^{\prime}\right\}$ as $\Omega^{\prime}$ ), the groups

$$
G_{i} \text { with } i \in \beta \stackrel{\text { def }}{=} \omega^{\prime} \cap\left(\bigcup\left(\Omega^{\prime} \backslash\left\{\omega^{\prime}\right\}\right)\right)
$$

freely generate their free product in the group $G_{\Omega^{\prime} \backslash\left\{\omega^{\prime}\right\}}$. But according to condition (**), the same groups $G_{i}$ with $i \in \beta$ freely generate their free product in the group $G_{\omega^{\prime}}$ (because $\omega^{\prime}$ contains an element $m$ not lying in $\beta$ ). Therefore, the group $G_{\Omega^{\prime}}$ decomposes into the amalgamated free product of $G_{\Omega^{\prime} \backslash\left\{\omega^{\prime}\right\}}$ and $G_{\omega^{\prime}}$ with amalgamated subgroup $\underset{i \in \beta}{*} G_{i}$.

The groups $G_{i}$ with $i \in \alpha$ lie in the factor $G_{\Omega^{\prime} \backslash\left\{\omega^{\prime}\right\}}$. Therefore, the assertion of Lemma 1 follows from the induction hypothesis applied to the set $\omega$ and the family $\Omega^{\prime} \backslash\left\{\omega^{\prime}\right\}$ as $\Omega^{\prime}$.

Case 2: $\omega \notin \Omega^{\prime}$. In this case, the proof is similar. We again use induction on the cardinality of $\Omega^{\prime}$. If $\Omega^{\prime}=\varnothing$, then we have nothing to prove. Suppose that $\left|\Omega^{\prime}\right| \geqslant 1$. In this case, according to conditions 1), 2), and 3), the family $F=\Omega^{\prime} \cup\{\omega\}$ contains a set $\omega^{\prime} \neq \omega$ with an element $m \in \omega^{\prime}$ not lying in $\bigcup\left(F \backslash\left\{\omega^{\prime}\right\}\right)$ (see Fig. 1). 


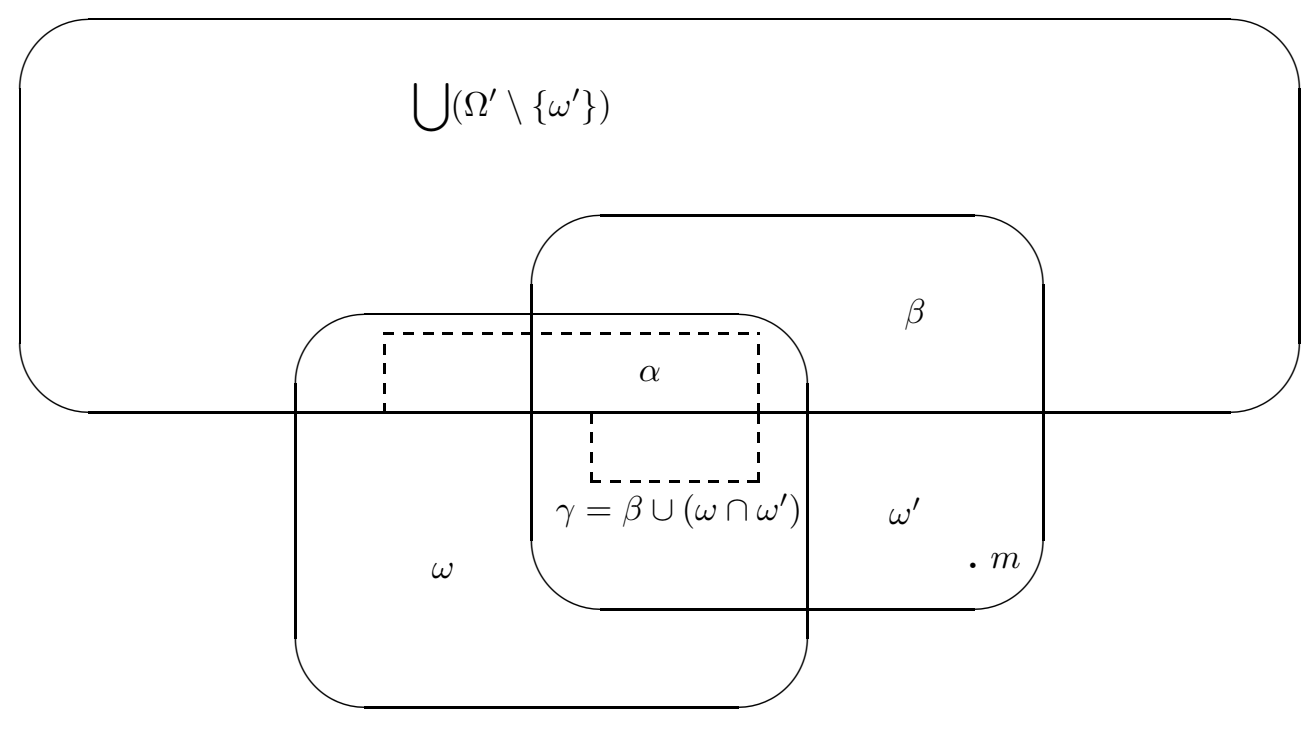

Fig. 1

By the induction hypothesis (applied to the set $\omega^{\prime}$ as $\omega$ and the family $\Omega^{\prime} \backslash\left\{\omega^{\prime}\right\}$ as $\Omega^{\prime}$ ), the groups

$$
G_{i} \text { with } i \in \beta \stackrel{\text { def }}{=} \omega^{\prime} \cap\left(\bigcup\left(\Omega^{\prime} \backslash\left\{\omega^{\prime}\right\}\right)\right)
$$

freely generate their free product in the group $G_{\Omega^{\prime} \backslash\left\{\omega^{\prime}\right\}}$. Therefore, the groups

$$
G_{i} \text { with } i \in \gamma \stackrel{\text { def }}{=} \beta \cup\left(\omega \cap \omega^{\prime}\right)=\omega^{\prime} \cap\left(\bigcup\left(\left(\Omega^{\prime} \cup \omega\right) \backslash\left\{\omega^{\prime}\right\}\right)\right)
$$

freely generate their product in the group

$$
H=\left(\underset{j \in\left(\omega \cap \omega^{\prime}\right) \backslash \beta}{*} G_{j}\right) * G_{\Omega^{\prime} \backslash\left\{\omega^{\prime}\right\}} .
$$

But condition (**) implies that the same groups $G_{i}$ with $i \in \gamma$, freely generate their free product in $G_{\omega^{\prime}}$ (because $\omega^{\prime}$ contains an element $m$ not lying in $\gamma$ ). Therefore, the group $G_{\Omega^{\prime}}$ decomposes into the amalgamated free product of the groups $H$ and $G_{\omega^{\prime}}$ :

$$
G_{\Omega^{\prime}}=H \underset{\left\langle G_{i} ; i \in \gamma\right\rangle}{*} G_{\omega^{\prime}} .
$$

The groups $G_{i}$ with $i \in \alpha$ lie in the factor $H$. Therefore, by the induction hypothesis applied to the set $\omega$ and the family $\Omega^{\prime} \backslash\left\{\omega^{\prime}\right\}$ as $\Omega^{\prime}$, the groups $G_{i}$ with $i \in \alpha \cap\left(\bigcup\left(\Omega^{\prime} \backslash\left\{\omega^{\prime}\right\}\right)\right.$ freely generate their free product in $G_{\Omega^{\prime} \backslash\left\{\omega^{\prime}\right\}}$. This immediately implies that the groups $G_{i}$ with subscripts $i \in \alpha$ freely generate their free product in $H$ and, hence, in the group $G_{\Omega^{\prime}}$ containing $H$ as a subgroup. Lemma 1 is proven.

Proof of Proposition 2. Clearly, it is sufficient to prove Proposition 2 for a finite family $\Omega$ of cardinality larger than one. In this case,

$$
G_{I}=G_{\Omega} *\left(\begin{array}{cc}
* & G_{i} \\
i \notin \bigcup \Omega
\end{array}\right),
$$

and $G_{\Omega}$ decomposes into the amalgamated free product:

$$
G_{\Omega}=G_{\omega_{\min }} \underset{K}{*} G_{\Omega \backslash\left\{\omega_{\min }\right\}},
$$

where the amalgamated subgroup $K$ is (by virtue of Lemma 1) the free product of the groups $G_{i}$ with $i \in \omega_{\min } \cap$ $\bigcup\left(\Omega \backslash\left\{\omega_{\min }\right\}\right)$. An obvious inductive argument completes the proof. 


\section{Proof of Theorem 2. The nonsplitting case}

In this section we prove Theorem 2 in the case when the group $\left\langle\left\{t_{i}\right\}\right\rangle \subseteq T$ is noncyclic.

Take an arbitrary countable group $S$. Put $t=\prod t_{i}$ and let us decompose $T$ into the union of cosets:

$$
T=\coprod_{x \in T /\langle t\rangle} c_{x}\langle t\rangle, \quad \text { where } c_{1}=1
$$

For each $x \in T /\langle t\rangle$, consider an isomorphic copy $G^{\left(c_{x}\right)}$ of the group $G$ assuming that the isomorphism maps $g \in G$ to $g^{\left(c_{x}\right)} \in G^{\left(c_{x}\right)}$. Let us rewrite the relation $\prod g_{i} t_{i}=1$ in the form

$$
t \prod_{i} g_{i}^{c_{x_{i}} t^{k_{i}}}=1
$$

Let $X_{1}$ be the set of all $x \in T /\langle t\rangle$ occurring in the reduced form of relation (2). Note that $\left|X_{1}\right| \geqslant 2$, because $\left\langle\left\{t_{i}\right\}\right\rangle \neq\langle t\rangle$ in the case under consideration. Put

$$
H_{1}=\underset{y \in X_{1}}{*} G^{\left(c_{y}\right)}
$$

and consider the unimodular relative presentation

$$
\widetilde{H}_{1}=\left\langle H_{1}, z \mid z \prod_{i} g_{i}\left(c_{x_{i}}\right) z^{k_{i}}=1\right\rangle
$$

over the group $H_{1}$. Theorem 3 implies that $\widetilde{H}_{1}$ has a quotient group $K_{1}$ such that

1) the group $S$ embeds into $K_{1}$;

2 ) in the group $K_{1}$, we have the decomposition

$$
\left\langle z,\left\{G^{\left(c_{y}\right)} ; y \in Y\right\}\right\rangle=\langle z\rangle_{\infty} *\left(\underset{y \in Y}{*} G_{y}\right)
$$

for each proper subset $Y \subset X_{1}$.

The group $K_{1}$ is a quotient of the group

$$
L_{1}=H_{1} *\langle z\rangle_{\infty}=\left(\underset{y \in X_{1}}{*} G^{\left(c_{y}\right)}\right) *\langle z\rangle_{\infty}
$$

by a normal subgroup $N_{1}$.

Now, consider the free product

$$
L=\left(\underset{y \in T /\langle t\rangle}{*} G^{\left(c_{y}\right)}\right) *\langle z\rangle_{\infty} .
$$

The group $T$ acts on the right on the group $L$ by automorphisms:

$$
z^{x}=z^{\varepsilon_{x}},\left(g^{\left(c_{y}\right)}\right)^{x}=g^{\left(c_{y x}\right) z^{l}},
$$

where $x \in T, y \in T /\langle t\rangle, \varepsilon_{x}= \pm 1$ depending on whether or not $x$ and $t$ commute, and the integer $l$ is uniquely determined from the equality $c_{y} x=c_{y x} t^{l}$.

For each $x \in T /\langle t\rangle$, consider the set $X_{x}=X_{1} x \subseteq T /\langle t\rangle$ and the subproduct

$$
L_{x}=\left(\underset{y \in X_{x}}{*} G^{\left(c_{y}\right)}\right) *\langle z\rangle_{\infty}
$$

of the free product $L$. The group $L_{x}$ has a normal subgroup $N_{x}=N_{1}^{x} \stackrel{\text { def }}{=} N_{1}^{\chi}$, where $\chi \in T$ is any representative of the element $x \in T /\langle t\rangle$.

Let us show that the family of subproducts $\left\{L_{x} \mid x \in T /\langle t\rangle\right\}$ together with the subgroups $N_{x} \triangleleft L_{x}$ satisfies the conditions of Proposition 2. Indeed, conditions 1), 2), and 3) of Proposition 2 follow directly from the strong uniqueproduct property of the group $T /\langle t\rangle$. Condition (**) for the pair $N_{1} \triangleleft L_{1}$ follows from decomposition (3). Condition (**) for any other pair $N_{x} \triangleleft L_{x}$ also holds, because the groups $L_{x}$ and $L_{1}$ are isomorphic and the isomorphism (the action 
of the element $x \in T$ ) maps the subgroup $N_{1}$ onto the subgroup $N_{x}$ and each factor $G^{\left(c_{y}\right)}$ of the group $L_{1}$ onto a subgroup $\left(G^{\left(c_{y x}\right)}\right)^{z^{l}}$ of $L_{x}$.

Thus, the conditions of Proposition 2 hold. Therefore, the natural mapping

$$
K_{x}=L_{x} / N_{x} \rightarrow K \stackrel{\text { def }}{=} L /\left\langle\left\langle\bigcup_{y \in T /\langle t\rangle} N_{y}\right\rangle\right\rangle
$$

is injective.

The group $T$ acts on $K$ by automorphisms. Take the corresponding semidirect product $T \curlywedge K$ and consider its quotient by the cyclic normal subgroup $\left\langle z t^{-1}\right\rangle$. The obtained group

$$
P=(T \curlywedge K) /\left\langle z t^{-1}\right\rangle
$$

is the required quotient group of $\widetilde{G}$.

Indeed, the group $G$ is embedded in $P$ as a subgroup: $G=G^{(1)} \subseteq K \subseteq P$. According to the definition of the action, we have $G^{\left(c_{x}\right)}=G^{c_{x}}$. Hence, the relations of the group $\widetilde{H}_{1}$ (which are valid in $K$ ) and the equality $t=z$ in $P$ give relation (2). Thus, $P=\langle T, G\rangle$ is a quotient group of $\widetilde{G}$ containing the subgroup $K_{1}$, which, in its turn, contains any given countable group $S$. This completes the proof of Theorem 2 in the case when the group $\left\langle\left\{t_{i}\right\}\right\rangle$ is noncyclic.

\section{Proof of Theorem 2. The splitting case}

Now, let us prove Theorem 2 in the case when the group $\left\langle\left\{t_{i}\right\}\right\rangle$ is cyclic. If presentation $(*)$ has the form $\widetilde{G}=$ $\langle G * T \mid t=1\rangle$, then the group $\widetilde{G}$ is the free product of two infinite groups $G$ and $T /\langle t\rangle$; therefore, it is SQ-universal (see [LS77]). In what follows, we assume that presentation $(*)$ has a different form.

The unimodularity condition implies that $\left\langle\left\{t_{i}\right\}\right\rangle=\langle t\rangle_{\infty}$, where $t=\prod t_{i}$. Consider the group $R$ with the unimodular relative presentation

$$
R=\left\langle G, z \mid \prod_{i=1}^{n} g_{i} z^{k_{i}}=1\right\rangle,
$$

where the exponents $k_{i}$ are determined from the equalities $t_{i}=t^{k_{i}}$. We have $k_{i} \neq 0$ and $\sum k_{i}=1$. It is known that the element $z$ has infinite order in the group $R$ and the equality $R=\langle z\rangle$ is valid only if $n=1$ and $G=\left\langle g_{1}\right\rangle$ [CR01]. In this case, Theorem 2 needs no proof. In other cases, take an element $r$ of the group $R$ not lying in $\langle z\rangle$, but such that $z^{k r} \neq z^{l}$ if the integers $k$ and $l$ are different. It is easy to see that such an element exists. Indeed, for all $x \in R$ let $k(x)$ be the non-negative integer defined by the equality $\langle z\rangle^{x} \cap\langle z\rangle=\left\langle z^{k(x)}\right\rangle$. There are three possible cases:

1) $k(x)=0$ for some $x \in R$;

2) $k(x)>1$ for some $x \in R$;

3) $k(x)=1$ for all $x \in R$.

In the first case we can take $r=x$. In the second case we can take $r=z^{x^{-1}}$. In the case 3), the cyclic subgroup $\langle z\rangle$ is normal in $R$ and does not coincides with its centraliser (because the index of this centraliser in $R$ is at most two and any virtually cyclic torsion-free group is cyclic); in this case, any element of the centraliser of $z$ not lying in $\langle z\rangle$ can be taken as $r$.

Let $\left\{t_{i j} ; i=1,2, \ldots, j=1, \ldots, 1000\right\}$ be an infinite sequence of elements of the centraliser of $t$ in $T$ such that all $t_{i j}^{ \pm 1}$ lie in different cosets by the normal subgroup $\langle t\rangle$. Such a sequence exists, because $T /\langle t\rangle$ is a nontrivial torsion-free group and the index of the centraliser of $t$ in $T$ is at most two. Take an arbitrary countable group $S=\left\{s_{1}, s_{2}, \ldots\right\}$ and put $K=R \times S$. Consider the group

$$
L=(K \underset{z=t}{*} T) /\left\langle\left\langle s_{i} \prod_{j=1}^{1000} r t_{i j} ; i=1,2, \ldots\right\rangle\right\rangle .
$$

This presentation of the group $L$ satisfies the small cancellation condition $C^{\prime}(1 / 100)$ for free amalgamated products (see [LS77]). Therefore, the natural mapping $S \rightarrow K \rightarrow L$ is injective. To complete the proof, it remains to note that the group $L$ is a quotient of $\widetilde{G}=R * T$. 


\section{Proof of Theorem 3}

Lemma 2. No infinite noncyclic group can be a union of a finite number of its cyclic subgroups.

Proof. Suppose that a group $G$ is a finite union of cyclic subgroups. Obviously, such a group has the property that any two infinite sets $X, Y \subseteq G$ contain a pair of commuting elements $x \in X, y \in Y$.

It is well known that all infinite groups with this property are abelian.*) Since any subgroup and any quotient group of the group $G$ is also a union of a finite number of cyclics, it is sufficient to show that $G \neq \mathbb{Z} \oplus \mathbb{Z}_{p}$. But the group $\mathbb{Z} \oplus \mathbb{Z}_{p}$ has infinitely many maximal cyclic subgroups: $\langle(1,1)\rangle,\langle(p, 1)\rangle,\left\langle\left(p^{2}, 1\right)\right\rangle, \ldots ;$ therefore, this group cannot be a finite union of cyclics.

Lemma 3. Suppose that $l \geqslant 2$ is an integer, $G_{1}, \ldots, G_{l}$ are noncyclic infinite groups, $u_{1}, \ldots, u_{s} \in G_{1} * \ldots * G_{l}$, and $S$ is a countable group. Then the group $G_{1} * \ldots * G_{l}$ has a normal subgroup $N$ such that

1) the group $S$ embeds into $\left(G_{1} * \ldots * G_{l}\right) / N$;

2) $N \cap\left\langle u_{i}, G_{i_{1}}, \ldots, G_{i_{l-1}}\right\rangle=\{1\}$ for all $i \in\{1, \ldots, s\}$ and $i_{1}, \ldots, i_{l-1} \in\{1, \ldots, l\}$.

Proof. Let $U \subseteq G_{1} \cup \ldots \cup G_{l}$ be the finite set consisting of all coefficients of all words $u_{i}$. Lemma 2 implies that the set

$$
M_{k}=G_{k} \backslash\left(\bigcup_{u \in U}\langle u\rangle\right)
$$

is infinite for each $k \in\{1, \ldots, l\}$. Consider the countable set of words

$$
v_{i}=\prod_{j=1}^{2017} \prod_{k=1}^{l} g_{i j k} \in G_{1} * \ldots * G_{l},
$$

where $g_{i j k} \in M_{k}$ and all $g_{i j k}^{ \pm 1}$ are different. Suppose that $S=\left\{s_{1}, s_{2}, \ldots\right\}$ and put

$$
H=\left(G_{1} * \ldots * G_{l} * S\right) /\left\langle\left\langle v_{1} s_{1}^{-1}, v_{2} s_{2}^{-1}, \ldots\right\rangle\right\rangle .
$$

Clearly, this presentation of the group $H$ satisfies the small cancellation condition $C^{\prime}(1 /(100 l)$ ) (see [LS77]). This implies that the natural mapping $S \rightarrow H$ is injective, and each word from the kernel $N$ of the natural epimorphism $G_{1} * \ldots * G_{l} \rightarrow H$ contains coefficients from each of the sets $M_{k}$. In particular, $N \cap\left\langle u_{i}, G_{i_{1}}, \ldots, G_{i_{l-1}}\right\rangle=\{1\}$, which completes the proof.

Now, let us prove Theorem 3. Suppose that the word $w$ has the form $u_{1} t^{\varepsilon_{1}} \ldots u_{s} t^{\varepsilon_{s}}$, where $u_{i} \in G_{1} * \ldots * G_{l}$ and $\varepsilon_{i} \in\{ \pm 1\}$. Note that, in the group $G_{1} * \ldots * G_{l}$, each nonidentity element $u_{i}$ has infinite order and is transcendental over each subgroup $G_{i_{1}} * \ldots * G_{i_{k}}$ not containing this element $u_{i}$.

Let us choose a normal subgroup $N \triangleleft G_{1} * \ldots * G_{l}$ according to Lemma 3 and put $G=\left(G_{1} * \ldots * G_{l}\right) / N$. Lemma 3 implies that the group $G$ contains any given countable group $S$ and the image of each nonidentity element $u_{i}$ in the group $G$ remains to be an element of infinite order transcendental over each subgroups $G_{i_{1}} * \ldots * G_{i_{k}}$ not containing this element $u_{i}$. To complete the proof, it remains to apply Proposition 1.

\section{Proof of Proposition 1}

We start with some simple facts.

Lemma 4. Let $u \in X * Y$ be an element of the free product of groups $X$ and $Y$ and suppose that $Z$ is a subgroup of $Y$ such that the element $u$ is algebraic (i.e., not transcendental) over $X * Z$. Then either $u \in(X * Z) Y(X * Z)$ or $u$ has the form $x_{1} u^{\prime} x_{2}$, where $x_{1}, x_{2} \in X * Z$ and the element $u^{\prime} \in X * Y$ has finite order.

We leave the proof of this elementary lemma to the readers as an easy exercise.

Lemma 5. Suppose that $A$ is a nontrivial subgroup of a group $B$ and $b \in B$. Then $b$ is transcendental over $A$ if and only if

$$
\left\langle\left\{A^{b^{i}} ; i \in \mathbb{Z}\right\}\right\rangle=\underset{i \in \mathbb{Z}}{*} A^{b^{i}}
$$

Proof. The "only if" part is obvious. To prove the "if" part, note that if $u \in A *\langle b\rangle_{\infty}$ is a nontrivial relation between $A$ and $b$ in the group $B$ and $a \in A \backslash\{1\}$, then $[a, u]$ is a nontrivial relation between the groups $A^{b^{i}}$. Lemma 5 is proven.

Throughout this section, we assume that a subgroup $H$ of a group $G$ satisfies conditions 1) and 2) from the definition of the strong magnusianity, presentation (1) is unimodular, and all nonidentity coefficients $g_{i}$ have infinite order in the group $G$. We have to prove that the element $t$ is transcendental over $H$ in the group $\widetilde{G}$.

*) This easily follows from a theorem of B. Neumann [Neu76] (the answer to a question of P. Erdős): The groups in which any infinite subset contains a pair of different commuting elements are precisely the groups with finite-index centres. 
Lemma 6. If

$$
\left\langle\left\{H^{t^{i}} ; i \in \mathbb{Z}\right\}\right\rangle=\underset{i \in \mathbb{Z}}{*} H^{t^{i}}
$$

in the group $\widetilde{G}$, then the element $t \in \widetilde{G}$ is transcendental over $H$.

Proof. If the group $H$ is nontrivial, the assertion immediately follows from Lemma 5 . If $H=\{1\}$, then the lemma asserts only that the element $t \in U$ has infinite order (if $w$ is not conjugate to $t^{ \pm 1}$ ); this fact was proven in [CR01].

Put

$$
\bar{H}=\underset{i \in \mathbb{Z}}{*} H_{i} \text { and } \bar{G}=\bar{H} \underset{H_{0}=H}{*} G
$$

where $H_{i}$ are isomorphic copies of the group $H$. Clearly, $\widetilde{G}$ has the presentation

$$
\widetilde{G} \simeq\left\langle\bar{G}, t \mid w(t)=1,\left\{H_{i}^{t}=H_{i+1} ; \quad i \in \mathbb{Z}\right\}\right\rangle .
$$

Let us move all letters $t^{ \pm 1}$ in the word $w$ to the left through the coefficients from $H$ by using the relations $H_{i} t^{ \pm 1}=$ $t^{ \pm 1} H_{i \pm 1}$. This reduces the presentation of $\widetilde{G}$ to the form

$$
\widetilde{G} \simeq\left\langle\bar{G}, t \mid \prod_{i=1}^{p} \bar{g}_{i} t^{k_{i}}=1,\left\{H_{i}^{t}=H_{i+1} ; i \in \mathbb{Z}\right\}\right\rangle
$$

where $k_{i} \in \mathbb{Z} \backslash\{0\}, \sum k_{i}=1, \bar{g}_{i} \in \bar{G}$, and each coefficient $\bar{g}_{i}$ has the form

$$
\bar{g}_{i}=\prod_{j=1}^{s} \bar{h}_{j} f_{j}, \quad \text { where } s \geqslant 1, f_{j} \in\left\{g_{1}, g_{2}, \ldots\right\} \backslash H, \bar{h}_{j} \in \bar{H} \backslash H \text { for } j \neq 1, \text { and } \bar{h}_{1} \in \bar{H} \text {. }
$$

Here $f_{j}, \bar{h}_{j}$, and $s$ depend on $i$. Note that $f_{1}, \ldots, f_{s}$ and $\bar{h}_{2}, \ldots, \bar{h}_{s}$ are transcendental over $H$ in $\bar{G}$.

If $p=1$, then the first relation of $\widetilde{G}$ can be rewritten in the form

$$
t=u, \quad \text { where } u=\bar{g}_{1}=\prod_{j=1}^{s} \bar{h}_{j} f_{j} \in \bar{G},
$$

and the whole presentation can be rewritten in the form

$$
\widetilde{G} \simeq\left\langle\bar{H} \underset{H_{0}=H}{*} G \mid\left\{H_{i}^{u}=H_{i+1} ; i \in \mathbb{Z}\right\}\right\rangle .
$$

By Lemma 6 , it is sufficient to prove the injectivity of the natural mapping $f: \bar{H} \rightarrow \widetilde{G}$.

If $s>1$, i.e., $u \notin \bar{H} G \bar{H}$, then the homomorphism $f$ is injective by virtue of Theorem 4 .

Now, suppose that $s=1$, i.e., $u=\bar{h}_{1} f_{1}$, where $\bar{h}_{1} \in \bar{H}$, and the element $f_{1} \in G$ is transcendental over $H$. In this case, the injectivity of the natural mapping $\bar{H}$ to $\widetilde{G}$ follows obviously from the decomposition

$$
\widetilde{G}=G \underset{K=L}{*}\left(H *\langle t\rangle_{\infty}\right),
$$

where $G \supseteq K=\left\langle f_{1}, H\right\rangle=\left\langle f_{1}\right\rangle_{\infty} * H \simeq L=\left\langle\left(\bar{h}_{1}\right)^{-1} t, H\right\rangle=\left\langle\left(\bar{h}_{1}\right)^{-1} t\right\rangle_{\infty} * H \subseteq H *\langle t\rangle_{\infty}$.

We proceed to the case $p>1$. Consider the following subgroups of $G *\langle t\rangle_{\infty}: G_{i}=t^{-i} G t^{i}, H_{i}=t^{-i} H t^{i}$,

$$
\bar{H}=\underset{i=-\infty}{*} H_{i}, \quad K^{(m)}=\underset{i=0}{*} G_{i}, \quad \text { and } \quad G^{(m)}=\bar{H} \underset{H_{0} * \ldots * H_{m}}{*} K^{(m)}
$$

Consider all possible expressions of the relation $w=1$ in the form

$$
c t \prod_{i=1}^{n} b_{i} t^{-1} a_{i} t=1, \quad \text { where } a_{i}, b_{i}, c \in G^{(m)} \text {. }
$$

Among all such expressions we choose those in which $m$ is minimal; after that, from all expressions with minimal $m$ we choose an expression with minimal $n$. For such a minimal expression (6), we have

1) $n \geqslant 1$ (i.e., the length of this expression is strictly larger than one); 
2) $a_{i} \notin G^{(m-1)}$ and $b_{i} \notin\left(G^{(m-1)}\right)^{t}$;

3) each coefficient $a_{i}$ is transcendental over $G^{(m-1)}$, and each coefficient $b_{i}$ is transcendental over $\left(G^{(m-1)}\right)^{t}$.

The first property follows from the inequality $p>1$, which implies that $m>0$ in any expression of length 1 ; therefore, $m$ can be decreased by replacing all occurrences of elements of $G_{m}$ by fragments of the form $t^{-1} g t$, where $g \in G_{m-1}$. The second property follows obviously from the minimality of $n$ and $m$. To prove property 3 ), we note that, in the normal form corresponding to the decomposition

$$
G^{(m)}=X * Y, \quad \text { where } X=\left(\underset{j \neq m}{*} H_{j}\right) * G_{0} * \ldots G_{m-1} \text { and } Y=G_{m}
$$

each $Y$-syllable of each coefficient $a_{i}$ lies in $\left(H\left\{f_{1}, \ldots, f_{p}\right\} H\right)^{t^{m}}$, and apply Lemma 4 putting $Z=H_{m}$. The transcendence of $b_{i}$ over $\left(G^{(m-1)}\right)^{t}$ is established similarly.

Now, suppose that the symbols $H_{i}$ and $G_{i}$ denote abstract isomorphic copies of the groups $H$ and $G$ and the groups $\bar{H}, K^{(m)}$, and $G^{(m)}$ are defined by formulae (5). Consider the following presentation of the group $\widetilde{G}$.

$$
\widetilde{G}=\left\langle G^{(m)}, t \mid c t \prod_{i=1}^{n} b_{i} a_{i}^{t}=1,\left\{G_{i}^{t}=G_{i+1} ; \quad i \in\{0, \ldots m-1\}\right\},\left\{H_{i}^{t}=H_{i+1} ; \quad i \in \mathbb{Z}\right\}\right\rangle .
$$

To complete the proof of Proposition 1, it remains to note that properties 1) and 3) of presentation (7) imply the injectivity of the natural mapping $G^{(m)} \rightarrow \widetilde{G}$ by virtue of the following theorem.

Theorem ([K193], see also [Fer96]). Suppose that $M$ and $N$ are isomorphic subgroups of a group $L, \varphi: M \rightarrow N$ is an isomorphism, $n \geqslant 1, a_{1}, \ldots, a_{n}$ are elements of $L$ transcendental over $M, b_{1}, \ldots, b_{n}$ are elements of $L$ transcendental over $N$, and $c \in L$. Then the system of equations

$$
\left\{\begin{array}{l}
x^{-1} g x=g^{\varphi}, \quad g \in M \\
c x \prod_{i=1}^{n} b_{i} x^{-1} a_{i} x=1
\end{array}\right.
$$

is solvable over $L$, i.e., the natural mapping $L \rightarrow\langle L, x \mid(* * *)\rangle$ is injective.

Applying this theorem to $L=G^{(m)}$ and $M=G^{(m-1)}$, we see that the natural mapping $\bar{H} \subset G^{(m)} \rightarrow \widetilde{G}$ is injective and the element $t \in \widetilde{G}$ is transcendental over $H$ (by Lemma 6 ).

\section{Proof of Theorem 4}

Let us reformulate Theorem 4 in terms of equations over groups. Recall that an equation over a group $G$ with unknown (or variable) $t$ is a formal expression of the form

$$
g_{1} t^{\varepsilon_{1}} g_{2} t^{\varepsilon_{2}} \ldots g_{n} t^{\varepsilon_{n}}=1
$$

where $g_{i} \in G$ and $\varepsilon_{i} \in \mathbb{Z}$. Equation (8) is said to be solvable over the group $G$ if there exists an overgroup $\widetilde{G}$ of $G$ and an element $\widetilde{t} \in \widetilde{G}$ (called a solution to equation $(*)$ ) such that $g_{1} \widetilde{t}^{\varepsilon_{1}} g_{2} \widetilde{t}^{\varepsilon_{2}} \ldots g_{n} \widetilde{t}^{\varepsilon_{n}}=1$ in $\widetilde{G}$. The notion of solvability of a system of equations with several unknowns over a group $G$ is defined similarly.

Lemma 7. Suppose that $G=A * B$ is the amalgamated free product of groups $A$ and $B$ with amalgamated subgroup $C, v=b_{0} a_{0} \ldots b_{m} a_{m} b_{m+1} \stackrel{C}{\in} G, \varphi$ is an automorphism of $B$, and

$$
\widehat{G}=\left\langle\underset{C}{A \underset{C}{*} B} \mid\left\{b^{v}=b^{\varphi} \mid b \in B\right\}\right\rangle \text {. }
$$

Then the injectivity of the natural mappings $A \rightarrow \widehat{G} \leftarrow B$ is equivalent to the solvability of the following system of equations with unknowns $t$ and $x$ over the group $G$ :

$$
\left\{\begin{array}{l}
b^{-x} b^{\varphi}=1 \quad \text { for } b \in B \backslash\{1\} \\
{[t, c]=1 \quad \text { for } c \in C \backslash\{1\}} \\
x^{-1} b_{0} a_{0}^{t} \ldots b_{m} a_{m}^{t} b_{m+1}=1
\end{array}\right.
$$


Proof. If $\widetilde{t}, \widetilde{x} \in \widetilde{G} \supseteq G$ is a solution to system (9), then the mapping $a \mapsto a^{\widetilde{t}}, b \mapsto b$ extends to a homomorphism $\widehat{G} \rightarrow \widetilde{G}$ injective on $A$ and on $B$. Conversely, suppose that the natural mappings $A \rightarrow \widehat{G} \leftarrow B$ are injective and consider an isomorphic copy

$$
\bar{G}=\left\langle\underset{\bar{C}}{\underset{\bar{C}}{*} \bar{B}} \mid\left\{\bar{b}^{\bar{v}}=\bar{b}^{\bar{\varphi}} \mid b \in B\right\}\right\rangle
$$

of the group $\widehat{G}$. It is easy to see that the elements $\widetilde{t}$ and $\widetilde{x}=b_{0} a_{0}^{\widetilde{t}} \ldots b_{m} a_{m}^{\widetilde{t}} b_{m+1}=\bar{v}$ of the HNN-extension

$$
\left\langle G \underset{B=\bar{B}}{*} \bar{G}, \widetilde{t} \mid\left\{a^{\widetilde{t}}=\bar{a} ; a \in A\right\}\right\rangle
$$

are solutions to system (9). This proves Lemma 7.

This lemma shows that Theorem 4 is implied by the following theorem.

Theorem $4^{\prime}$. Suppose that $G$ is a group with subgroups $C, B$, and $B^{\varphi}, \varphi: B \rightarrow B^{\varphi}$ is an isomorphism, $m \geqslant 1$, $b_{i}, a_{i} \in G$, and the elements $a_{0}, \ldots, a_{m}$ and $b_{1}, \ldots, b_{m}$ are transcendental over $C$. Then system (9) is solvable over $G$.

To prove Theorem 4', we need Howie's lemma. For simplicity, we formulate this lemma in a special case related to system (9).

Consider a map (tessellation) on an oriented two-dimensional sphere. The corners of this map are labelled by elements of the group $G$. The edges are directed (the directions are shown by arrows on the figures) and labelled by the variables $t$ and $x$.

The label of a vertex in such a situation is defined as the product of the labels of all corners near this vertex listed clockwise. The label of a vertex is an element of $G$ defined up to conjugation. For example, the label of the vertex shown in Figure 2 is $a_{0}^{-1} c a_{0}^{2} c^{\prime}$.

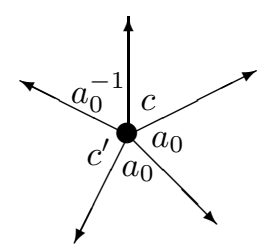

Fig. 2

To obtain the label of a face, we should walk along its boundary anticlockwise and write down the labels of all its corners and edges; the label of an edge should be written as its inverse if we walk through it against the arrow. The label of a face is an element of the group $G * F(t, x)$ (the free product of $G$ and the free group with basis $\{t, x\}$ ), defined up to a cyclic permutation. For example, the label of the upper left face shown in Figure 3 is $b^{-x} b^{\varphi}$.

Such a labelled map is called a spherical Howie diagram (or simply diagram) over system (9) if

1) one vertex is distinguished and called the exterior vertex, the other vertices are called interior;

2) the label of each interior vertex is the identity element of $G$;

3) the label of each face is either the left-hand side of an equation of system (9) or the word inverse to the left-hand side of an equation of system (9); all possible types of faces are shown in Figure 3, where the letters $b$ and $c$ denote any nonidentity elements of the groups $B$ and $C$, respectively, and the edges not labelled by $x$ are assumed to be labelled by $t$ (the numbers written outside the faces should be ignored for a while). 

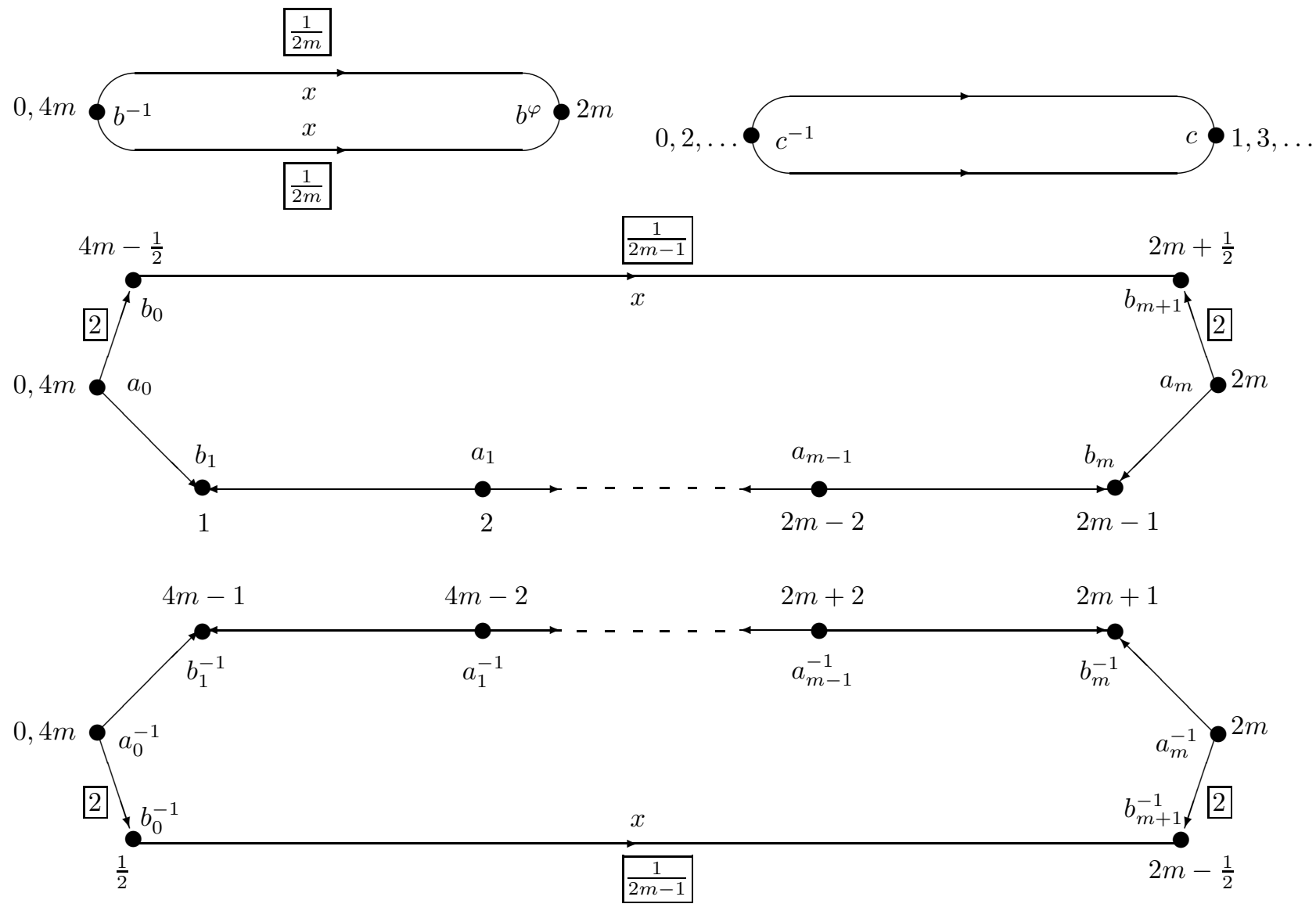

Fig. 3

A Howie diagram is called reduced if it has no edge $e$ such that the two faces containing $e$ are different and their labels written starting with the edge $e$ are mutually inverse; such a pair of faces with a common edge is called a reducible pair.

Lemma 8 [How83]. System (9) is not solvable over the group $G$ if and only if there exists a spherical diagram over this system such that the label of its exterior vertex is not the identity element of $G$. Any such diagram with minimal number of faces is reduced.

We say that a diagram over system (9) is strongly reduced if it is reduced and different faces labelled by $b^{-x} b^{\varphi}$ or $[c, t]$ have no common edges.

Lemma 9. Any spherical diagram with minimal number of faces such that the label of its exterior vertex is a nonidentity element is strongly reduced.

Proof. Indeed, if some diagram has a pair of faces labelled by, e.g., $b^{-x} b^{\varphi}$ and $\left(b^{\prime}\right)^{-x}\left(b^{\prime}\right)^{\varphi}$ and having a common edge, then either this pair is a reducible pair or we can erase the common edge multiplying the labels of the corresponding corners (Fig. 4) and obtain a diagram fewer faces and the same label of the exterior vertex. This implies the nonminimality of the initial diagram and proves the lemma.
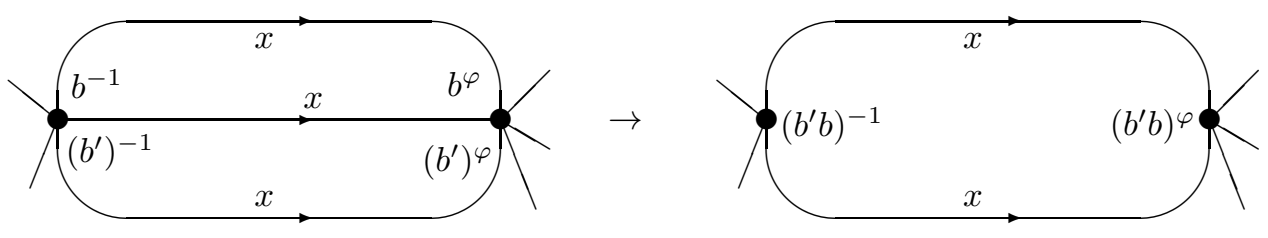

Fig. 4

Now, suppose that, on the contour of each face $D$ of a map on the sphere, there is a moving point (a car) $\alpha_{D}$. The car $\alpha_{D}$ moves continuously anticlockwise (i.e., the interior of the face $D$ remains on the left from the car) without U-turns, stops, and "infinite decelerations", that is, it covers each edge in a finite time. We call such a motion regular. 
If the number of cars being at a moment $\tau \in \mathbb{R}$ at a point $p$ of the sphere equals the multiplicity of this point (in other words, either two cars simultaneously pass the same internal point $p$ of an edge at the moment $\tau$ or there are $k$ cars at a vertex $p$ of degree $k$ at the moment $\tau$ ), then we say that a complete collision occurs at the point $p$ at the moment $\tau$; the point $p$ is called a point of complete collision. Figure 5 shows complete collisions on an edge and at a vertex of degree three.
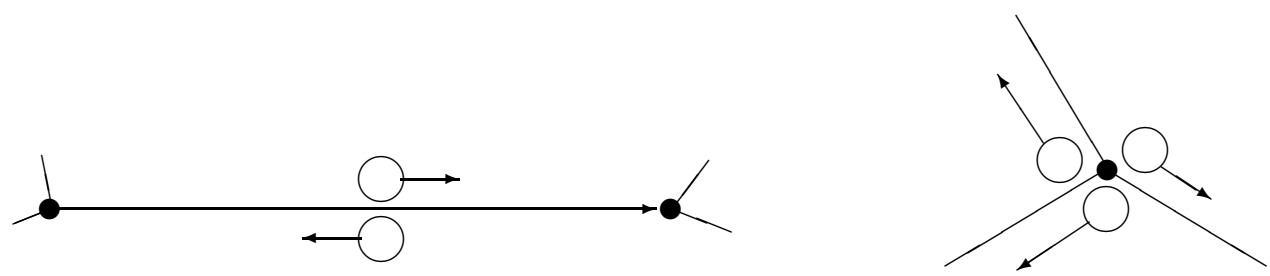

Fig. 5

Lemma 10 [K193] (see also [FeR96]). Any regular motion on a sphere has at least two points of complete collision.

Now, take a spherical diagram over presentation (9) as a map and consider the following regular motion on this map:

a) the car going around a face labelled by $b^{-x} b^{\varphi}$ moves anticlockwise uniformly with speed $\frac{1}{2 m}$ edges per unit time and visits the corner labelled by $b^{-1}$ at time zero.

b) the car going around a face labelled by $[t, c]$ moves anticlockwise uniformly with unit speed (one edge per unit time) and visits the corner with label $c^{-1}$ at time zero;

c) the car going (anticlockwise) around a face labelled by $x^{-1} b_{0} a_{0}^{t} \ldots b_{m} a_{m}^{t} b_{m+1}$ is at the corner labelled by $a_{0}$ at time zero; then it goes along $2 m$ edges labelled by $t$ with unit speed and arrives at the moment $2 m$ in the corner labelled by $a_{m}$; then, the car goes along the next edge labelled by $t$ with speed 2 and the edge labelled by $x$ with speed $\frac{1}{2 m-1}$; after that, it moves along the edge labelled by $t$ with speed 2 and returns at the moment $4 m$ to the initial corner labelled by $a_{0}$; then, the motion is repeated with period $4 \mathrm{~m}$;

d) the car moving (anticlockwise) around a face labelled by $\left(x^{-1} b_{0} a_{0}^{t} \ldots b_{m} a_{m}^{t} b_{m+1}\right)^{-1}$ is at the corner labelled by $a_{0}^{-1}$ at time zero; then it goes along the first edge labelled by $t$ with speed 2 and along the next edge labelled by $x$ with speed $\frac{1}{2 m-1}$; after that, the car goes along the edge labelled by $t$ with speed 2 and arrives at the moment $2 m$ in the corner labelled by $a_{m}^{-1}$; then, the car goes along the next $2 m$ edges labelled by $t$ with unit speed and returns at the moment $4 m$ to the initial corner labelled by $a_{0}^{-1}$; then the motion is repeated with period $4 m$.

This motion is regular and periodic with period $4 m$ (on the faces labelled by [t, $c$, the minimal period is 2 ). Figure 3 shows the detailed schedule of this motion during the interval $0 \leqslant \tau \leqslant 4 m$; the boxed numbers near edges indicate the speed of the car on these edges (by default, the speed is 1).

Lemma 11. For the motion on a strongly reduced diagram over system (9) described above, the complete collisions can occur only at the exterior vertex.

\section{Proof.}

A collision on an edge labelled by $t$ at time $\tau$ means that, at this moment, the direction of the motion of one of the cars coincides with the direction the edge, while the direction of the motion of the other colliding car is opposite to the direction of this edge (Fig. 5, left). But the schedule of the motion is such that, at each moment $\tau$, either all cars being on the edges labelled by $t$ move in the direction of the edge (this is so when the integer part of $\tau$ is even) or all cars being on edges labelled by $t$ move in the direction opposite to the direction of the edge (this is so when the integer part of $\tau$ is odd).

A similar argument shows that cars cannot collide on an edge labelled by $x$ : during the time intervals $[0,2 m]+4 m \mathbb{Z}$, all cars being on edges labelled by $x$ move in the direction of the edge, and during the time intervals $[2 m, 4 m]+4 m \mathbb{Z}$, all cars being on edges labelled by $x$ move in the direction opposite to the direction of the edge.

Thus, collisions can occur only at vertices.

A complete collision at the start or the end vertex of an edge labelled by $x$ cannot occur, because the diagram is strongly reduced and, hence, each edge labelled by $x$ separates a face labelled by $b^{-x} b^{\varphi}$ and a face labelled $\left(x^{-1} b_{0} a_{0}^{t} \ldots b_{m} a_{m}^{t} b_{m+1}\right)^{ \pm 1}$ (see Fig. 6). This means that one car visits the start vertex of an edge labelled by $x$ at the moments $4 m \mathbb{Z}$, and another car visits this vertex at the moments $4 m \mathbb{Z} \pm \frac{1}{2}$. Thus, a complete collision cannot occur. For similar reasons, a complete collision cannot occur at the end vertex of an edge labelled by $x$ : one car visits such a vertex at the moments $4 m \mathbb{Z}+2 m$ and another, at the moments $4 m \mathbb{Z}+2 m \pm \frac{1}{2}$. 


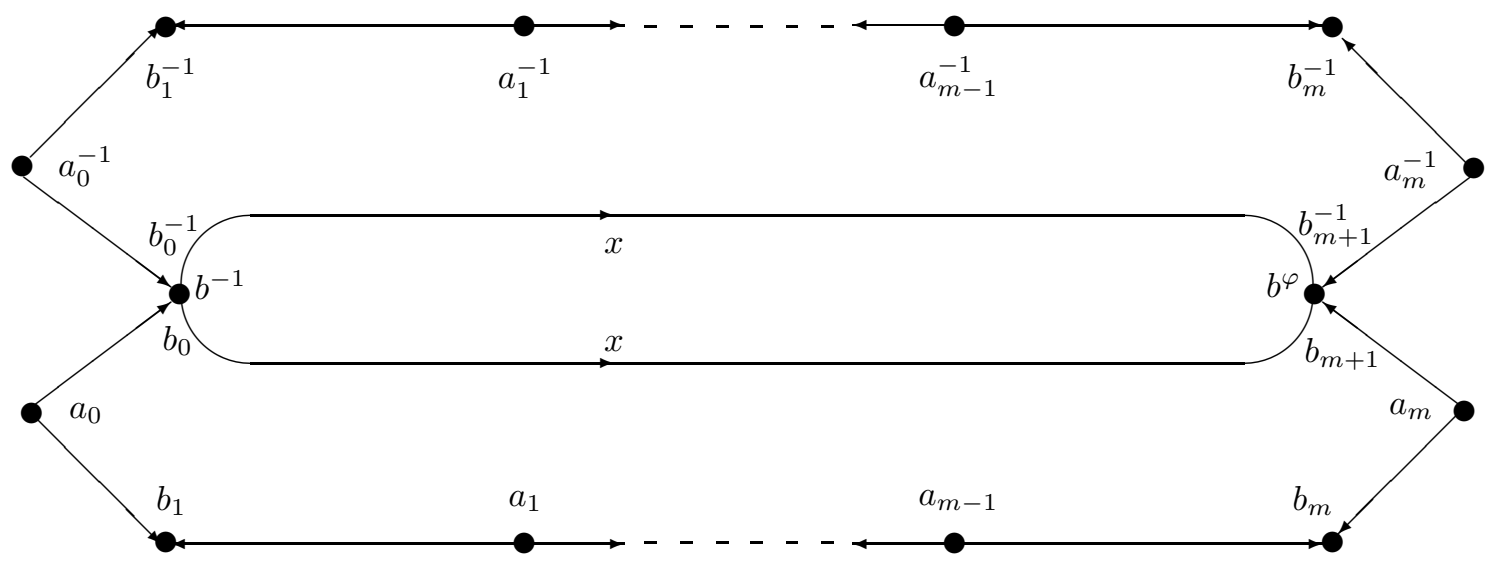

Fig. 6

A complete collision at an interior vertex being neither the start nor the end of an edge labelled by $x$ cannot occur too. Such vertices are visited only at integer moments of time and, at every such moment $\tau$, each car being at such a vertex is either at a corner labelled by $c \in C \backslash\{1\}$ or at a corner labelled by $d \in\left\{a_{0}^{ \pm 1}, b_{1}^{ \pm 1}, \ldots, b_{m}^{ \pm 1}, a_{m}^{ \pm 1}\right\}$. Moreover, if at the moment $\tau$ a car is at a corner labelled by $d$ and, simultaneously, another car is at a corner labelled by $d^{\prime}$, where $d, d^{\prime} \in\left\{a_{0}^{ \pm 1}, b_{1}^{ \pm 1}, \ldots, b_{m}^{ \pm 1}, a_{m}^{ \pm 1}\right\}$, then either $d^{\prime}=d$ or $d^{\prime}=d^{-1}$. $)^{\prime}$ This means that a vertex of complete collision occurring at the moment $\tau$ has label of the form $\prod z_{i}$, where $z_{i} \in\left\{d, d^{-1}\right\} \cup C \backslash\{1\}$. Since the diagram is reduced, $d$ and $d^{-1}$ cannot be neighbours in the sequence $\left(z_{i}\right)$. Since the diagram is strongly reduced, two elements from $C \backslash\{1\}$ cannot be neighbours in this sequence either. Therefore, the label $\prod z_{i}$ of the vertex of complete collision cannot be the identity element of $G$, because the element $d$ is transcendental over $C$. Hence, the vertex of complete collision cannot be an interior vertex of the diagram. Figure 2 shows a hypothetical vertex at which a complete collision occurs at the moment $\tau=0$. This vertex cannot be interior, because $a_{0}^{-1} c a_{0}^{2} c^{\prime} \neq 1$ in the group $G$. This completes the proof of Lemma 11.

Theorem $4^{\prime}$ follows easily from lemmata 8-11. Indeed, suppose that system (9) is unsolvable. Then, by Lemma 8 , there exists a diagram over this system. By Lemma 9, this diagram can be assumed strongly reduced. By Lemma 11, it admits a regular motion with at most one point of complete collision, which contradicts Lemma 10. This proves Theorems $4^{\prime}$ and 4 .

\section{REFERENCES}

[B84] Brodskii S.D. Equations over groups and one-relator groups // Sib. Mat. Zh. 1984. T.25. no.2. P.84-103.

[K194] Klyachko Ant.A. The Kervaire-Laudenbach Conjecture and Equations over Groups, Cand. Sci. Dissertation, Moscow: MSU, 1994.

[K105] Klyachko Ant.A. The Kervaire-Laudenbach conjecture and presentations of simple groups // Algebra i Logika. 2005. T. 44. no.4. P. 399-437. See also arXiv:math.GR/0409146.

[Kl06a] Klyachko Ant.A. How to generalize known results on equations over groups // Mat. Zametki. 2006. T.79. no.3. P.409-419. See also arXiv:math.GR/0406382.

[K106b] Klyachko Ant.A. Free subgroups of one-relator relative presentations // Algebra i Logika (to appear). See also arXiv:math.GR/0510582.

[LS77] Lyndon R.C., Schupp P.E. Combinatorial Group Theory, Springer-Verlag, Berlin/Heidelberg/New York, 1977.

[Lo86] Lossov K.I. SQ-universality of free products with finite amalgamated subgroups // Sib. Mat. Zh. 1986. T.27. no.6. P.128-139.

[Ols95] Olshanskii A.Yu. SQ-universality of hyperbolic groups // Mat. Sbornik. 1995. T.186. no.8. P.119-132.

[AMO06] Arzhantseva G., Minasyan A., Osin D. The SQ-universality and residual properties of relatively hyperbolic groups // arXiv:math.GR/0601590.

[BaPr78] Baumslag B., Pride S. Groups with two more generators than relators // J. London Math. Soc. 1978. V.17. P.425-426.

[Bu05] Button J.O. Large mapping tori of free group endomorphisms // arXiv:math.GR/0511715.

[CR01] Cohen M.M., Rourke C. The surjectivity problem for one-generator, one-relator extensions of torsion-free groups // Geometry \& Topology. 2001. V.5. P.127-142. See also arXiv:math.GR/0009101.

[Ed84] Edjvet M. Groups with balanced presentations // Arch. Math. 1984. V.42. no.4. P.311-313.

*) The latter can occur only at the moments $\tau \in\{0,2 m\}+4 m \mathbb{Z}$. Thus, $d=a_{0}^{ \pm 1}$ or $d=a_{m}^{ \pm 1}$. This makes it possible to slightly weaken the conditions of Theorems 4 and $4^{\prime}$, but we do not need such a weakening here. 
[FeR96] Fenn R., Rourke C. Klyachko's methods and the solution of equations over torsion-free groups // L'Enseignment Mathématique. 1996. T.42. P.49-74.

[FoR05] Forester M., Rourke C. Diagrams and the second homotopy group // Comm. Anal. Geom. 2005. V.13. P.801-820. see also arXiv:math.AT/0306088.

[Gr83] Gromov M. Volume and bounded cohomology // Inst. Hautes Etudes Sci. Publ. Math. 1982. No. 56. P.5-99. (1983).

[How83] Howie J. The solution of length three equations over groups // Proc. Edinburgh Math. Soc. 1983. V.26. P.89-96.

[How98] Howie J. Free subgroups in groups of small deficiency // J. Group Theory. 1998. V.1. no. 1. P.95-112.

[K193] Klyachko Ant.A. A funny property of a sphere and equations over groups // Comm. Algebra. 1993. V.21. P.25552575.

[La05] Lackenby M. A characterisation of large finitely presented groups // J. Algebra. 2005. V.287. no.2. P. $458-473$. See also arXiv:math.GR/0403129.

[Neu76] Neumann B.H. A problem of Paul Erdős on groups // J. Austral. Math. Soc. Ser. A. 1976. V.21. no.4. P.467-472.

[Neu73] Neumann P.M. The SQ-universality of some finitely presented groups // J. Austral. Math. Soc. 1973. V.16. P.1-6.

[OlOs06] Olshanskii A.Yu., Osin D.V. Adding high-powered relations to large groups: A short proof of Lackenby's result // arXiv:math.GR/0601589.

[P88] Promyslow S.D. A simple example of a torsion free nonunique product group // Bull. London Math. Soc. 1988. V.20. P.302-304.

[RS87] Rips E., Segev Y. Torsion free groups without unique product property // J. Algebra 1987. V.108. P.116-126.

[SaSc74] Sacerdote G.S., Schupp P.E. SQ-universality in HNN groups and one relator groups // J. London Math. Soc. 1974. V.7. P.733-740.

[Stö83] Stöhr R. Groups with one more generator than relators // Math. Z. 1983. V.182. no. 1. P.45-47. 